\title{
Fibronectin peptides in cell migration and wound repair
}

\author{
Kenneth M. Yamada \\ Craniofacial Developmental Biology and Regeneration Branch, National Institute of Dental and Craniofacial \\ Research, National Institutes of Health, Building 30, Room 421, 30 Convent Drive MSC 4370, \\ Bethesda, Maryland 20892-4370, USA. \\ Phone: (301) 496-9124; Fax: (301) 402-0897; E-mail: Kenneth.Yamada@nih.gov.
}

Defective healing of chronic wounds is a serious clinical problem; for example, decubitus and leg ulcers afflict roughly 5 million people in the US alone. Normal wound repair depends on molecules like fibronectin to promote cell adhesion and migration (1). This large adhesive glycoprotein provides a crucial substrate for many forms of cell migration, such as in embryonic migratory pathways and in the provisional matrix of healing wounds. Besides acting as a substrate, fibronectin also has certain proteolytic fragments that can promote chemotactic migration (e.g., see ref. 2), and fibronectin or its fragments can also activate integrin signaling (reviewed in refs. 3, 4). Because fibronectin augments cell migration, ideas for stimulating wound repair have included soaking wound dressings in fibronectin, providing fibronectin in eye drops, and developing synthetic polymeric substrates that contain biologically active fragments of the fibronectin sequence.

A novel approach reported by Livant et al. in this issue of JCI (5) involves stimulating wound repair with a specific fibronectin peptide. This study reports intriguing findings that differ conceptually from the prior literature on fibronectin peptides. It describes a fibronectin peptide with unexpectedly high apparent affinity, an unusual mechanism of action, and high efficacy in vivo. These results appear to challenge current thinking in the field but, if correct, could lead to novel therapies for wounds.

Fibronectin has nearly a dozen regions termed "cell-binding sites" that can be recognized and bound by cell-surface integrins (e.g., see ref. 6). These sites, including the four examples shown in Figure 1, can be reproduced as biologically active short peptides. When presented to cells as a peptide, a cell-binding site of fibronectin can function in several different ways (Figure 2): (a) It can mimic the intact protein as a substrate for migration. For example, if the peptide is adsorbed to a surface or attached covalently to a carrier molecule, it can be bound by a cell-surface integrin, which can mediate direct cell adhesion or cell migration. (b) The peptide can serve as a competitive inhibitor. When added in solution, the functional peptide can bind to the receptor and competitively inhibit binding of an adhesion molecule, thereby blocking adhesion and migration. (c) Binding of fibronectin peptides can augment integrin signaling (7) and can induce expression of collagenase or activate cell-to-cell adhesiveness in particular cell types (8-10). These peptides are generally thought to mimic activity seen in the intact fibronectin molecule, and their activities are generally significantly lower than those of the parent protein.
A dream for many in the field has been to apply fibronectin peptides, which can be synthesized and potentially combined in various ways to generate specificity, to wound repair and tissue engineering. Conversely, inhibitory peptides could also be used to block unwanted processes such as tumor cell invasion and metastasis (11). Conventional thought in this field has focused on the use of fibronectin or its mimetics as adhesive and migration substrates in wounds (Figure 2, left). A very different approach is proposed by Livant and coworkers in this issue of the JCI (5). These authors focused on the PHSRN (Pro-His-Ser-Arg-Asn) sequence of fibronectin, because their initial antibody inhibition studies suggested a role for this sequence in activating cell invasion. PHSRN had been characterized previously as a "synergy" sequence that cooperated with the RGD sequence in mediating effective inte-

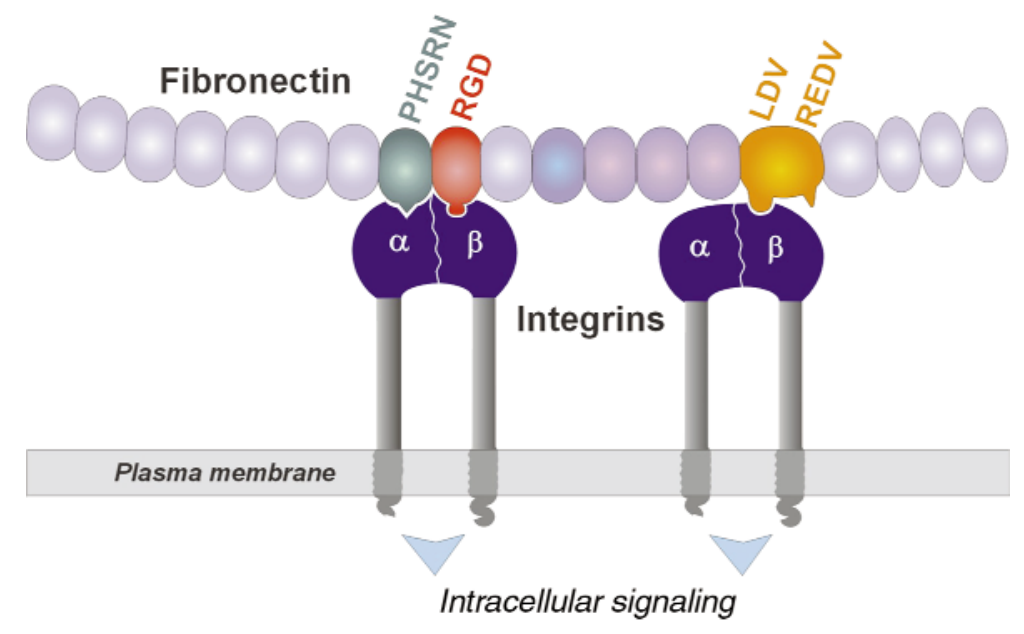

Figure 1

The fibronectin molecule consists of repeating globular domains with specific cell-binding sites for interactions with integrins. Four of the fibronectin peptide sites (abbreviated using the single-letter code for amino acids) that can interact with specific integrins are shown extending down toward the integrins. Integrins contain one $\alpha$ and one $\beta$ subunit, which span the plasma membrane and terminate in short cytoplasmic domains that are thought to mediate intracellular signaling. 


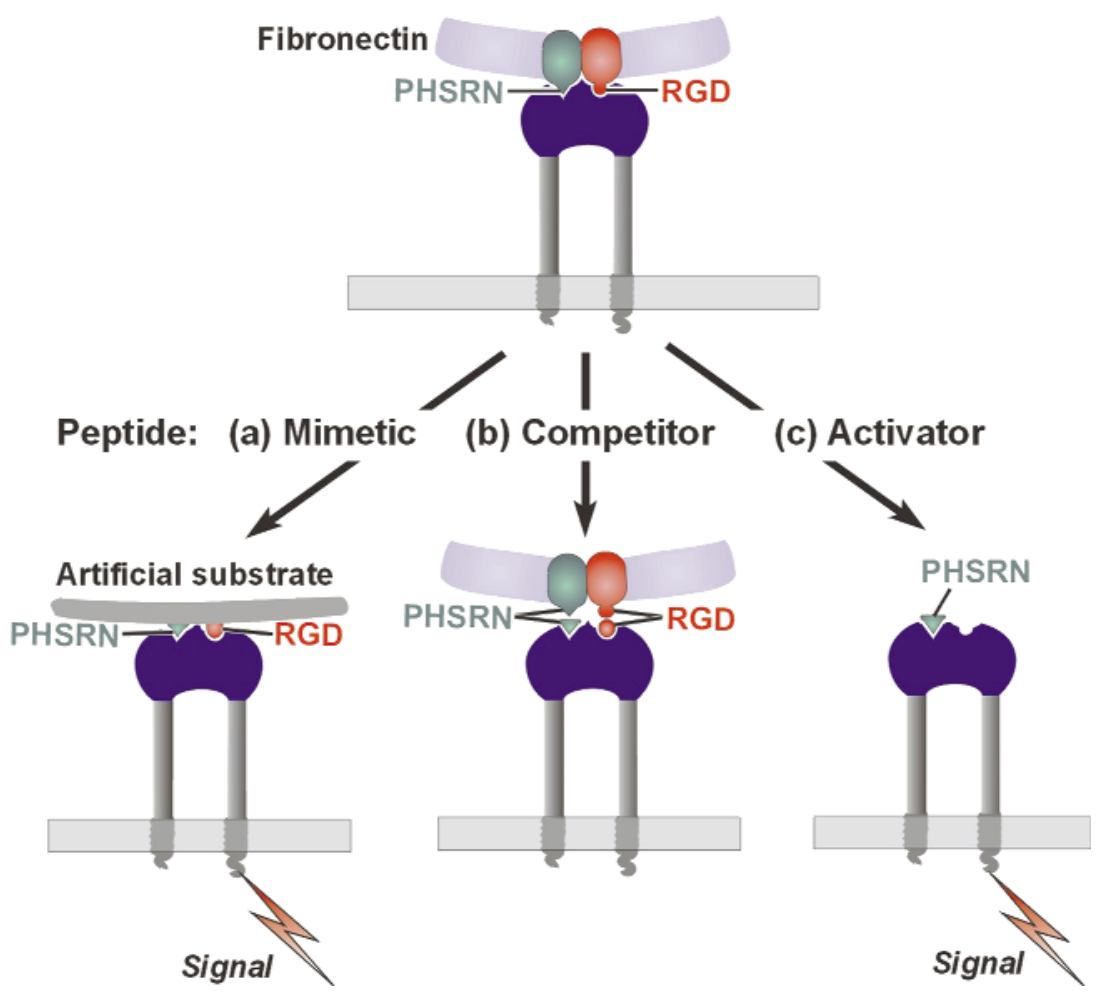

Figure 2

Synthetic fibronectin peptides such as PHSRN could function in at least three different ways: (a) by providing binding sites for specific integrins, e.g., using peptides covalently attached to a synthetic matrix; (b) by competing for the binding of native fibronectin by integrins; or (c) by directly activating an integrin.

grin-mediated cell adhesion and migration (12-14). In contrast to conventional thinking, Livant et al. (5) report that this fibronectin peptide has remarkably potent activity in triggering invasion of fibroblasts or keratinocytes across a heterologous (invertebrate-derived) basement membrane. Moreover, low doses of the peptide activate wound repair in vivo in a murine model. Chemically blocking the charged $\mathrm{NH}_{2}$ - and $\mathrm{COOH}$-termini of the peptide (which helps peptides better mimic a sequence within a polypeptide chain) resulted in the highest activity. These findings contrast markedly with those in earlier studies, which demonstrated much lower activity for PHSRN peptides in inhibiting cell adhesion competitively or in mediating direct adhesion (14). These earlier studies were consistent with the dogma that PHSRN represents a minimal version of the overall synergy region, whereas the present study reports peptide activities more than tenfold higher than even the original protein (5), suggesting that cells can bind PHSRN with much higher healing in a genetically obese diabetic mouse model. They report that a single treatment with $2 \mu \mathrm{g}$ of the short AcPHSRN-NH ${ }_{2}$ peptide dramatically stimulates repair, accelerating impaired closure of 4-mm punch biopsy wounds from $20-42$ days to only 8 days. This peptide, but not a randomized version, enhanced keratinocyte and fibroblast migration into the wounds and improved vascularization. Treatment with this peptide also slightly accelerated wound closure in the control heterozygous mice. Whether similar enhancement of wound repair will be seen in other wound healing systems remains to be determined.

Interestingly, this group has also recently shown that a similar peptide, modified by an arginine $\rightarrow$ cysteine substitution to generate an inhibitor, reversed tumor metastases in rats (17). These striking stimulatory and inhibitory results with PHSRN derivatives in vivo contrast with the general experience that small peptides are not ideal candidates for therapeutics because they diffuse rapidly and undergo prompt renal clearance, as found for another fibronectin peptide (18). However, it is possible that a dry wound might retain peptides; in this regard, tracer studies on the half-life of the PHSRN peptides in wounds will be quite informative. Other questions for the future include establishing which integrin is involved in vivo, the nature of any signaling pathways activated by this reagent, the mechanism by which cell migration is stimulated, and the significance of heightened migration or invasion for the reported effects in accelerating wound repair.

Because these imaginative studies by Livant and coworkers $(5,17)$ challenge orthodoxy and report such impressive acceleration of wound healing (and reversal of metastasis) in animal models, they will arouse considerable interest and, possibly, controversy. Nevertheless, the authors seem to have made a good case for their novel approaches, and it is now up to the field to test them. It is important to establish promptly whether these potentially exciting results represent a major breakthrough or a temporary detour in the quest for creative therapies to alleviate clinical problems in wound repair.

\footnotetext{
1. Clark, R.A.F. 1996. The molecular and cellular biology of wound repair. Plenum Press. New York, New York, USA. 611 pp.

2. Clark, R.A., Wikner, N.E., Doherty, D.E., and Norris, D.A. 1988. Cryptic chemotactic activity of
} 
fibronectin for human monocytes resides in the $120-\mathrm{kDa}$ fibroblastic cell-binding fragment. J. Biol. Chem. 263:12115-12123.

3. Schwartz, M.A., Schaller, M.D., and Ginsberg, M.H. 1995. Integrins: emerging paradigms of signal transduction. Annu. Rev. Cell Dev. Biol. 11:549-599.

4. Giancotti, F.G., and Ruoslahti, E. 1999. Integrin signaling. Science. 285:1028-1032.

5. Livant, D.L., et al. 2000. The PHSRN sequence induces extracellular matrix invasion and accelerates wound healing in obese diabetic mice. $J$. Clin. Invest. 105:1537-1545

6. Yamada, K.M. 1999. Cell adhesion molecules. In Encyclopedia of molecular biology. T.E. Creighton, editor. John Wiley \& Sons Inc. New York, New York, USA. 361-366.

7. Miyamoto, S., Akiyama, S.K., and Yamada, K.M. 1995. Synergistic roles for receptor occupancy and aggregation in integrin transmembrane function. Science. 267:883-885.

8. Werb, Z., Tremble, P.M., Behrendtsen, O., Crowley, E., and Damsky, C.H. 1989. Signal transduc- tion through the fibronectin receptor induces collagenase and stromelysin gene expression. J. Cell Biol. 109:877-889.

9. Lash, J.W., Linask, K.K., and Yamada, K.M. 1987. Synthetic peptides that mimic the adhesive recognition signal of fibronectin: differential effects on cell-cell and cell-substratum adhesion in embryonic chick cells. Dev. Biol. 123:411-420.

10. Monier-Gavelle, F., and Duband, J.L. 1997. Cross talk between adhesion molecules: control of $\mathrm{N}$ cadherin activity by intracellular signals elicited by $\beta 1$ and $\beta 3$ integrins in migrating neural crest cells. J. Cell Biol. 137:1663-1681.

11. Humphries, M.J., Olden, K., and Yamada, K.M. 1986. A synthetic peptide from fibronectin inhibits experimental metastasis of murine melanoma cells. Science. 233:467-470.

12. Obara, M., Kang, M.S., and Yamada, K.M. 1988. Site-directed mutagenesis of the cell-binding domain of human fibronectin: separable, synergistic sites mediate adhesive function. Cell. 53:649-657.

13. Bowditch, R.D., et al. 1991. Integrin alpha IIb beta
3 (platelet GPIIb-IIIa) recognizes multiple sites in fibronectin. J. Biol. Chem. 266:23323-23328.

14. Aota, S., Nomizu, M., and Yamada, K.M. 1994. The short amino acid sequence Pro-His-Ser-ArgAsn in human fibronectin enhances cell-adhesive function. J. Biol. Chem. 269:24756-24761.

15. Lane, T.F., Iruela-Arispe, M.L., Johnson, R.S., and Sage, E.H. 1994. SPARC is a source of copperbinding peptides that stimulate angiogenesis. $J$. Cell Biol. 125:929-943.

16. Malinda, K.M., et al. 1999. Identification of laminin alpha 1 and beta 1 chain peptides active for endothelial cell adhesion, tube formation, and aortic sprouting. FASEB J. 13:53-62.

17. Livant, D.L., et al. 2000. Anti-invasive, antitumorigenic, and antimetastatic activities of the PHSCN sequence in prostate carcinoma. Cancer Res. 60:309-320.

18. Humphries, M.J., Yamada, K.M., and Olden, K. 1988. Investigation of the biological effects of anti-cell adhesive synthetic peptides that inhibit experimental metastasis of B16-F10 murine melanoma cells. J. Clin. Invest. 81:782-790. 